\title{
Hybrid Bead Air Filters with Low Pressure Drops at a High Flow Rate for the Removal of Particulate Matter and HCHO
}

\author{
Hee Ju Kim ${ }^{1}$, Ye Jin Kim ${ }^{1}$, Yu Jin Seo ${ }^{1}$, Ji Hee Choi ${ }^{1}$, Hye Young Koo ${ }^{2}$ and Won San Choi ${ }^{1, *}$ (D) \\ 1 Department of Chemical and Biological Engineering, Hanbat National University, 125 Dongseodaero, \\ Yuseong-gu, Daejeon 305-719, Korea; kimhj0924@naver.com (H.J.K.); agoqkfkrl@naver.com (Y.J.K.); \\ $6285999 @$ naver.com (Y.J.S.); wlgml1350@naver.com (J.H.C.) \\ 2 Functional Composite Materials Research Center, Jeonbuk Institute of Advanced Composite Materials, \\ Korea Institute of Science and Technology (KIST), 92 Chudong-ro, Bongdong-eup, Wanju-gun, \\ Seoul 136-791, Korea; koohy@kist.re.kr \\ * Correspondence: choiws@hanbat.ac.kr; Tel.: +82-42-821-1540
}

check for updates

Citation: Kim, H.J.; Kim, Y.J.; Seo, Y.J.; Choi, J.H.; Koo, H.Y.; Choi, W.S. Hybrid Bead Air Filters with Low Pressure Drops at a High Flow Rate for the Removal of Particulate Matter and HCHO. Polymers 2022, 14, 422. https://doi.org/10.3390/ polym14030422

Academic Editor: Maria Graça Rasteiro

Received: 19 November 2021 Accepted: 13 January 2022 Published: 21 January 2022

Publisher's Note: MDPI stays neutral with regard to jurisdictional claims in published maps and institutional affiliations.

Copyright: (C) 2022 by the authors. Licensee MDPI, Basel, Switzerland. This article is an open access article distributed under the terms and conditions of the Creative Commons Attribution (CC BY) license (https:// creativecommons.org/licenses/by/ $4.0 /)$.

\begin{abstract}
A tower air filtration system was designed in which bead air filters (BAFs) were actively rotated by a fan motor to remove particulate matter (PM) or HCHO gas. Three types of BAF, hydrophilic, hydrophobic, and hybrid, were prepared and compared for the removal of PM and HCHO gas. A tower air filtration system loaded with hybrid BAFs purified $3.73 \mathrm{~L}$ of PM $\left(2500 \mu \mathrm{g} / \mathrm{m}^{3} \mathrm{PM}_{2.5}\right)$ at a high flow rate of $3.4 \mathrm{~m} / \mathrm{s}$ with high removal efficiency $\left(99.4 \%\right.$ for $\left.\mathrm{PM}_{2.5}\right)$ and a low pressure drop (19 Pa) in $6 \mathrm{~min}$. Against our expectations, the $\mathrm{PM}_{2.5}$ removal efficiency slightly increased as the air velocity increased. The hybrid BAF-200 showed excellent recyclability up to 50 cycles with high removal efficiencies (99.4-93.4\% for $\mathrm{PM}_{2.5}$ ). Furthermore, hydrophilic BAF-200 could permanently remove $3.73 \mathrm{~L}$ of $\mathrm{HCHO}$ gas $(4.87 \mathrm{ppm})$ and return the atmosphere to safe levels $(0.41-0.31 \mathrm{ppm})$ within 60 min without any desorption of $\mathrm{HCHO}$ gas.
\end{abstract}

Keywords: particulate matters; air filters; $\mathrm{HCHO}$; removal efficiency; pressure drop

\section{Introduction}

When particulate matter (PM)-laden air passes through a fixed air filter, the PM is filtered, and a pressure drop occurs because the fixed air filter acts as a barrier to the incoming air. The pressure drop is inevitably generated by the fixed air filter during air filtration. The minimization of this pressure drop is one of the major challenges for ideal air filters because a high pressure drop causes high energy consumption. Thus, various air filters have been developed to address the pressure drop [1-4]. However, most of the abovementioned air filters are still fixed, regardless of the structure and function of the air filter. A lottery draw machine-inspired movable air filter system that deviates from typical air filters was recently reported for the removal of PM [5]. This air filter showed excellent removal efficiency and an extremely low pressure drop because of its unique air filter structure. However, the development of novel air filters that are not fixed is still attractive.

PM is a hydrophilic chemical mixture consisting of nitrate, sulfate, silicate, chloride, and elemental carbon, which are generally formed by burning fossil fuels [1]. Therefore, much effort has been devoted to developing highly polar superhydrophilic or hydrophilic air filters [6-13]. Relatively little attention has been focused on hybrid air filters composed of hydrophilic and hydrophobic materials. Only a few groups have focused on hybrid air filters with enhanced removal performance under high relative humidity and general conditions [14-16]. The role of hydrophobicity in hybrid air filters has not been extensively studied.

Formaldehyde (HCHO) is another target for removal by air filters. It is a highly toxic gas emitted from many industrial products. Humans who are frequently exposed to formaldehyde are diagnosed with cancer at higher rates than people who are not as 
exposed $[17,18]$. The concentration of $\mathrm{HCHO}$ in the air is sometimes higher when air filters are used because air filters are unable to filter $\mathrm{HCHO}$ but are still generally used in enclosed spaces such as homes, offices, hospitals, and laboratories [10,11]. Plasma catalysis, thermal oxidation, and catalytic oxidation have been proposed to decompose $\mathrm{HCHO}$ into $\mathrm{CO}_{2}$ and $\mathrm{H}_{2} \mathrm{O}$. However, these methods inevitably produce toxic byproducts, such as $\mathrm{NO}_{\mathrm{x}}, \mathrm{O}_{3}$, and $\mathrm{OH}$ radicals, and consume large amounts of energy [19-22]. Although the adsorption method is environmentally friendly and economical, $\mathrm{HCHO}$ removal by adsorption on air filters has not been extensively studied. Thus, it is of great significance to develop multifunctional air filters with high removal efficiency and a low pressure drop for the removal of PM and HCHO.

Herein, we report a tower air filtration system in which bead air filters (BAFs) are actively rotated by a fan motor to remove PM or HCHO gas. Three types of BAFs, hydrophilic, hydrophobic, and hybrid BAFs, are prepared and compared for the removal of $\mathrm{PM}$ and $\mathrm{HCHO}$ gas.

\section{Experimental Section}

\subsection{Materials}

Sulfate (melamine-formaldehyde sponge) MFS and polydimethylsiloxane (PDMS) (Sylgard 184) were purchased from BASF and Dow Chemical, respectively. Polyvinyl alcohol (PVA) (Mw: 130,000 Da) and n-hexane $\left(\mathrm{C}_{6} \mathrm{H}_{14}, 95 \%\right)$ were purchased from SigmaAldrich. Konjac glucomannan (KGM) was purchased from Zhejiangs. All chemicals were used without further purification. Deionized (DI) water with a resistance of $18 \mathrm{M} \Omega \mathrm{cm}$ was obtained from a Millipore Simplicity 185 system.

\subsection{Preparation of Bead MFS}

Two hundred pieces of cylindrical MFS (diameter: $0.5 \mathrm{~cm}$ and height: $0.5 \mathrm{~cm}$ ) were added to a beaker containing DI water $(200 \mathrm{~mL})$. The inside of the beaker was coated with sandpaper, and the contents were stirred for $8 \mathrm{~h}$. The bead sponge was prepared by immersion of the cylindrical MFS into a beaker containing water, followed by stirring and collision of the MFS. The MFS collided with the inside wall of the beaker, which was wrapped in sandpaper. The cylindrical MFS was gradually transformed into a bead sponge by physically etching the MFS with the sandpaper. The resulting products were washed three times with DI water and dried in an oven at $50{ }^{\circ} \mathrm{C}$ for $4 \mathrm{~h}$.

\subsection{Preparation of Hydrophilic BAFs}

PVA powder $(1 \mathrm{~g})$ was dissolved in DI water $(100 \mathrm{~mL})$ under vigorous stirring at $200{ }^{\circ} \mathrm{C}$ for $2 \mathrm{~h}$. Then, KGM powder $(0.1 \mathrm{~g})$ was added and dissolved in the resulting solution at $100{ }^{\circ} \mathrm{C}$. Next, 100-200 MFS beads were immersed in the abovementioned PVA-KGM solution and heated in an oven at $100{ }^{\circ} \mathrm{C}$ for $3 \mathrm{~h}$. The final products were washed three times with DI water and dried in an oven at $50{ }^{\circ} \mathrm{C}$ for $4 \mathrm{~h}$.

\subsection{Preparation of Hydrophobic BAFs}

A PDMS solution (mass ratio) containing a cross-linker (1), prepolymer (10), and n-hexane (100) was prepared. Next, 100-200 MFS beads were immersed in the PDMS solution and heated in an oven at $100{ }^{\circ} \mathrm{C}$ for $1 \mathrm{~h}$. The final products were washed three times with ethanol and dried in an oven at $50{ }^{\circ} \mathrm{C}$ for $4 \mathrm{~h}$.

\subsection{Preparation of Hybrid BAFs}

Hybrid BAFs were prepared by a half-and-half mixture of hydrophilic and hydrophobic BAFs (hybrid BAF-n (n/2 MFS/PVA-KGM + n/2 MFS/PDMS).

\subsection{PM and VOC Filtration}

PM was generated by burning incense. The PM removal test chamber $(3.63 \mathrm{~L})$ was filled with low $\left(500 \mu \mathrm{g} / \mathrm{m}^{3}\right)$, medium $\left(1000 \mu \mathrm{g} / \mathrm{m}^{3}\right)$, and high $\left(2500 \mu \mathrm{g} / \mathrm{m}^{3}\right)$ concentrations 
of $\mathrm{PM}_{2.5}$. The air velocities generated by the fan motor were $2.4,3.4$, and $4.4 \mathrm{~m} / \mathrm{s}$. A constant air flow was injected into the air filter to rotate the BAFs. A handheld air quality detector was used to measure the PM concentrations before and after filtration. The removal efficiency was calculated by comparing the measured PM concentrations before and after filtration. All PM filtration tests were performed at relative humidity from $29 \%$ to $36 \%$ (standard deviation: 1.56). When air was injected into the chamber, the pressure drop was zero point-adjusted. After the addition of BAFs into the chamber and injection of air, the pressure drop was measured at the input and output sections by a differential pressure gauge. For removal of $\mathrm{HCHO}$ gas, the main chamber containing a tower air filtration system (middle) was connected to a glass vial (left) containing $\mathrm{HCHO}$ gas and a detector (right). An air velocity of $1.0 \mathrm{~m} / \mathrm{s}$ was injected into the glass vial $(50 \mathrm{~mL})$ containing $10 \mu \mathrm{L}$ of $\mathrm{HCHO}$ to push the $\mathrm{HCHO}$ gas from the left to the main chamber. The variation in the $\mathrm{HCHO}$ gas concentration emitted from tower air filtration system BAFs was monitored by an $\mathrm{HCHO}$ detector.

\subsection{Characterization}

SEM/EDX analyses were performed using a Hitachi S-4800 instrument. FT-IR spectra were obtained using a Sinco Nicolet IS5 instrument. TGA was performed using a thermogravimetric analyzer (Sinco TGA N-1500, Seoul, Korea) over a temperature range of $25-800{ }^{\circ} \mathrm{C}$ at a heating rate of $10{ }^{\circ} \mathrm{C} \mathrm{min}{ }^{-1}$ in air (flow rate, $60 \mathrm{~cm}^{2} \mathrm{~min}^{-1}$ ). The contact angle measurements were carried out using a contact angle meter (SEO Phoenix 300 Touch, Yongin-si, Korea) at ambient temperature, and the volume of the probing liquid was $20 \mu \mathrm{L}$. After 5 min of vigorous rotation of each BAF within the glass chamber, the surface potential of each sample was measured by an electrostatic voltmeter (HSK-5008 L, Sejong, Korea). A handheld air quality detector (HT-9601, Dongguan Xintai Instrument, Dongguan City, China) was used to measure the PM concentrations before and after filtration. By comparing the PM concentrations before and after filtration, the removal efficiency (RE) can be calculated according to the following equation:

$$
\operatorname{RE}(\%)=\left(C_{0}-C_{1}\right) / C_{0} \times 100 \%
$$

where $C_{0}\left(\mu \mathrm{g} \mathrm{m}^{-3}\right)$ and $C_{1}\left(\mu \mathrm{g} \mathrm{m}^{-3}\right)$ refer to the PM concentrations before and after filtration, respectively. The pressure drop was measured by a differential pressure gauge (TESTO 510i, TESTO, Titisee-Neustadt, Germany). The flow rate was measured by a flowmeter (TESTO 450i, TESTO, Germany). The error ranges of removal efficiency, pressure drop, and removal time were below $1.5 \%, 3 \%$, and $4 \%$, respectively. $\mathrm{HCHO}$ was measured by an HCHO measuring meter (BQ16, Trotec GmbH \& Co. KG, Heinsberg, Germany).

\section{Results and Discussion}

Figure 1a shows a schematic illustration of the synthesis of two types of BAF, hydrophilic and hydrophobic, through a physical etching of an MFS, followed by one-step coating with hydrophilic and hydrophobic polymers, respectively. Hydrophilic and hydrophobic BAFs were prepared by one-step coating of the bead sponge with a polymer solution of PVA/ KGM and PDMS, respectively. Hydrophilic, hydrophobic, or hybrid BAFs were loaded into the tower air filtration system (Figure 1b). A tower air filtration system in which hybrid BAFs are actively rotated to capture PM or HCHO is proposed.

Figure $2 a-d$ shows images of the shape variation of the cylindrical MFS while it was stirred in the beaker and wrapped in sand paper. As the stirring time increased, the sharp edge of the cylindrical MFS became round (Figure $2 b-d$ ). After $8 \mathrm{~h}$, the cylindrical MFS became almost perfectly spherical (Figure 2d). The size of the resulting MFS bead decreased to $80 \%$ of the original size of the cylindrical MFS (Figure $2 \mathrm{~b}, \mathrm{~d}$ ). The sizes of the MFS beads to be used as BAFs were controlled by varying the sizes of cylindrical MFSs. MFS beads with sizes of $4 \mathrm{~mm}$ and $9 \mathrm{~mm}$ were prepared and used in this study (Figure S1). Our physical etching method enabled the mass production of homogeneous MFS beads. After 
the formation of the MFS beads, PVA-KGM and PDMS were separately coated onto the MFS beads to synthesize hydrophilic and hydrophobic BAFs, respectively (Figure 2e,f).

a)

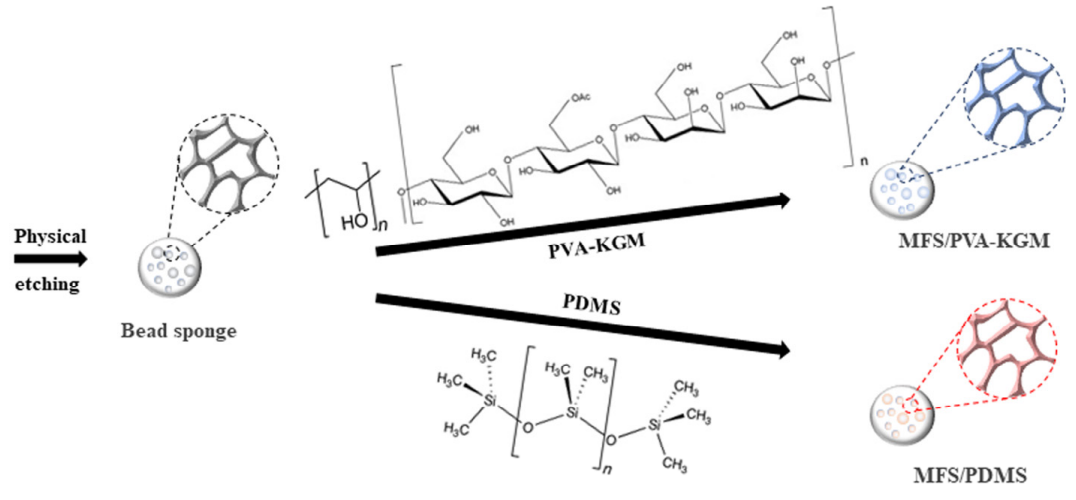

b)

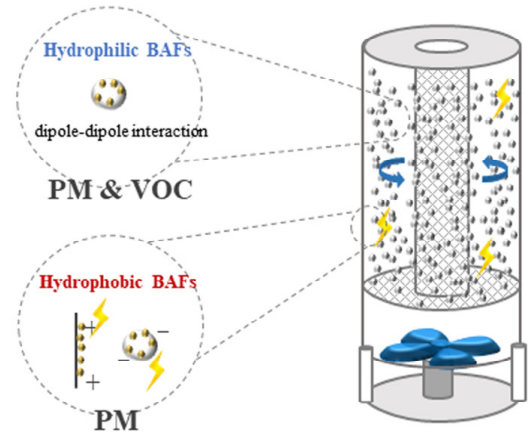

Figure 1. A schematic illustration of (a) the synthesis of two types of BAF, hydrophilic and hydrophobic, and (b) a tower air filtration system in which BAFs are actively rotated to capture PM or HCHO.

a)
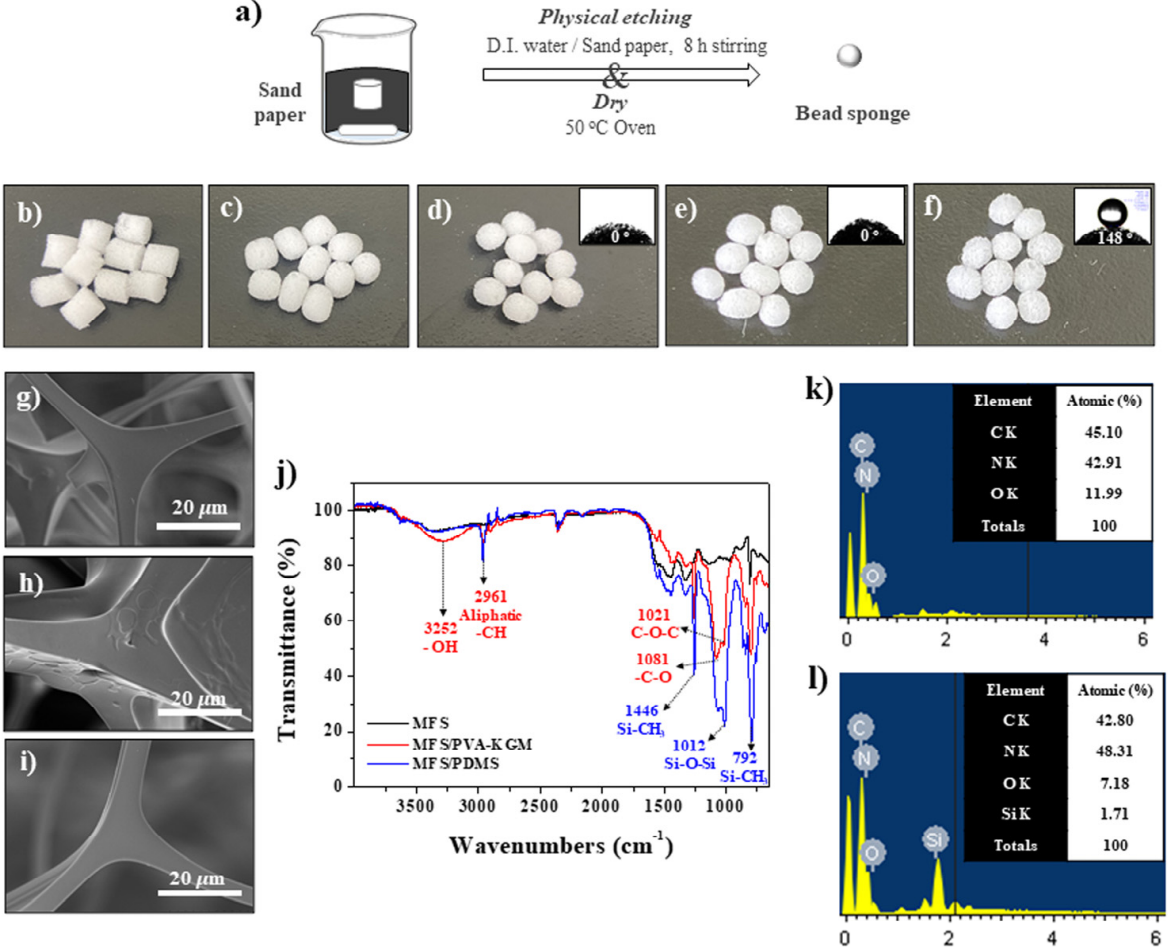

Figure 2. (a-d) Images showing the process of BAF formation with stirring time for (b) $0 \mathrm{~h}$, (c) $4 \mathrm{~h}$, and $8 \mathrm{~h}$. Images of (e) hydrophilic BAFs (MFS/PVA-KGM) and (f) hydrophobic BAFs (MFS/PDMS). The insets show the corresponding WCA data. SEM images of (g) MFS, (h) hydrophilic BAFs, and (i) hydrophobic BAFs. (j) FT-IR and EDX data of (k) hydrophilic and (1) hydrophobic BAFs. 
Figure $2 \mathrm{~g}-\mathrm{i}$ shows scanning electron microscopy (SEM) images of a sponge (MFS) bead, the hydrophilic BAF (MFS/PVA-KGM), and the hydrophobic BAF (MFS/PDMS). The sponge bead possessed an interconnected network skeleton with a smooth surface morphology (Figure 2g). The hydrophilic BAF (MFS/PVA-KGM) and hydrophobic BAF (MFS/PDMS) featured surface morphologies that were slightly more rough than that of the sponge bead, indicating that each polymer was coated on the sponge bead (Figure 2h,i). No detached fragments were observed upon handling each BAF. No color changes of hydrophilic and hydrophobic BAFs were observed after the coating of each polymer on the sponge bead (Figure 2d-f). The water contact angles (WCAs) of the sponge bead, hydrophilic BAF, and hydrophobic BAF were $0^{\circ}, 0^{\circ}$, and $148^{\circ}$, indicating that MFS, MFS/PVAKGM, and MFS/PDMS had superhydrophilic, superhydrophilic, and hydrophobic characteristics, respectively (insets of Figure $2 \mathrm{~d}-\mathrm{f}$ ). The absorption peaks at $3252 \mathrm{~cm}^{-1}(\mathrm{OH})$, $2961 \mathrm{~cm}^{-1}$ (aliphatic CH), $1081 \mathrm{~cm}^{-1}$ (C-O), and $1021 \mathrm{~cm}^{-1}$ (C-O-C), the characteristic groups in the PVA-KGM spectrum [23-25], were increased or newly formed due to the coating of PVA-KGM for hydrophilic BAF (MFS/PVA-KGM) (red line, Figure 2j). New absorption peaks at $1446 \mathrm{~cm}^{-1}\left(\mathrm{Si}_{-} \mathrm{CH}_{3}\right)$ and $1012 \mathrm{~cm}^{-1}$ (Si-O-Si) were observed for hydrophobic BAFs (MFS/PDMS) (blue line) [23-25]. The intensity of the peak at $792 \mathrm{~cm}^{-1}$ related to $\mathrm{Si}-\mathrm{CH}_{3}$ remarkably increased for hydrophobic BAFs (MFS/PDMS) (blue line) [23-25]. Energy dispersive X-ray (EDX) data also confirmed the synthesis of hydrophilic and hydrophobic BAFs (Figure 2k,l and Figure S2). The C content in the hydrophilic BAF was much higher than that in the hydrophobic BAF due to the PVA-KGM coating (Figure 2k). $\mathrm{Si}$ was observed in the hydrophobic BAF due to the PDMS coating (Figure 21).

A tower air filtration system was composed of two parts, including an air filter section containing BAFs (top) and a fan motor section (bottom) (Figure 3a). The air filter section consisted of an inner cylindrical frame (Cu mesh) and an outer chamber (glass). The BAFs can fly between the inner frame and outer chamber. A constant air flow was generated by the fan motor and injected into the air filter section to rotate the BAFs. A BAF with a diameter of $4 \mathrm{~mm}$ was used for the removal of PM. The tower air filtration system was placed in an acrylate chamber $(3.63 \mathrm{~L})$ containing a hazardous level of PM with $\mathrm{PM}_{2.5}$ concentrations higher than $2500 \mu \mathrm{g} / \mathrm{m}^{3}$. By fan motor, the PM-laden air was sucked into the bottom part of the air filtration system, passed through the filter part containing BAFs, purified, and emitted from the upper part of the air filtration system. The pressure drop was measured at input and output points by a differential pressure gauge (Figure S3). The unfiltered PM was detected in the right part of the chamber. The air velocities generated by the fan motor were $2.4-4.4 \mathrm{~m} / \mathrm{s}$, which were an order of magnitude higher than the previously reported values [12,26-28]. When a constant air flow was injected into the part of the air filter containing the BAFs, the BAFs vigorously moved everywhere within the inside of the air filter part, and this phenomenon was observed in all cases (Figure 3b).

Figure $3 \mathrm{c}$ shows the $\mathrm{PM}_{2.5}$ removal efficiencies of the air filtration systems loaded with different numbers of hydrophilic BAF (MFS/PVA-KGM) (100, 200, 300, and 400). The air filtration system using 100, 200, 300, or 400 BAFs was denoted BAF-100, 200, 300, or 400. The $\mathrm{PM}_{2.5}$ removal efficiencies of BAF-100 and BAF-200 increased, but those of BAF-300 and BAF-400 remained as analogous as that of BAF-200, which indicates that the mobilities of BAF-300 and BAF-400 were lower due to the increased bulkiness and volume (Figure 3c). In fact, the BAF-300 and BAF-400 were not uniformly dispersed within the chamber by the fan (Figure S4). The pressure drops of the BAFs increased as the number of BAFs increased due to the increased bulkiness and volume (Figure 3c). However, the pressure drop of the BAF-200, which featured the highest $\mathrm{PM}_{2.5}$ removal efficiency, was only $19 \mathrm{~Pa}$ at an air velocity of $3.4 \mathrm{~m} / \mathrm{s}$. Previously reported air filters feature much higher pressure drops than BAF-200 with values of 45-499 Pa, even at a much lower air velocity of $0.2 \mathrm{~m} / \mathrm{s}$. Because BAF-200 accounts for only $7.7 \%$ of the total volume of the air filter chamber, the injected air flow is seldom impeded by BAFs, causing the pressure drop to decrease. The time to reach the $\mathrm{PM}_{2.5}$ concentration below $50 \mu \mathrm{g} / \mathrm{m}^{3}$ was also measured for BAF-100, 200, 300, and 400. The removal time was $7 \mathrm{~min}$ for BAF-200 and 300 and $8 \mathrm{~min}$ 
for BAF-400 (Figure S5a). BAF-100 never reached a PM 2.5 concentration below $50 \mu \mathrm{g} / \mathrm{m}^{3}$ even after $10 \mathrm{~min}$. Considering the $\mathrm{PM}_{2.5}$ removal efficiency (99.2\%), pressure drop (19 $\mathrm{Pa}$ ), and removal time $(7 \mathrm{~min})$, BAF-200 was determined to be an optimized condition for hydrophilic BAFs.
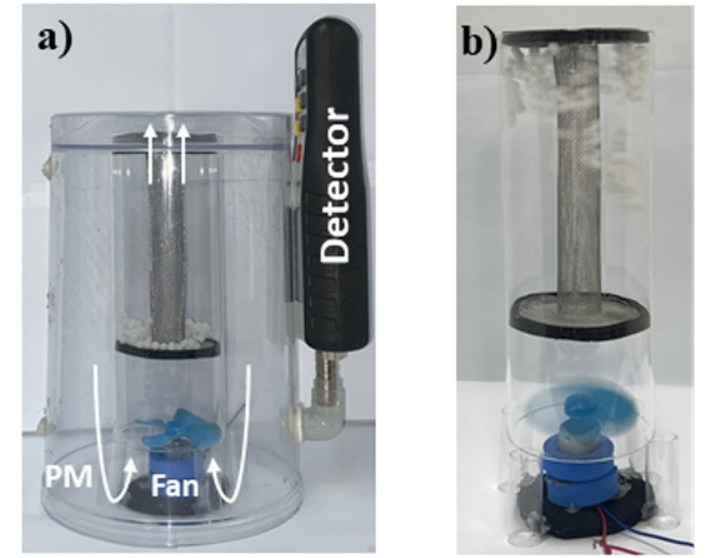

c)

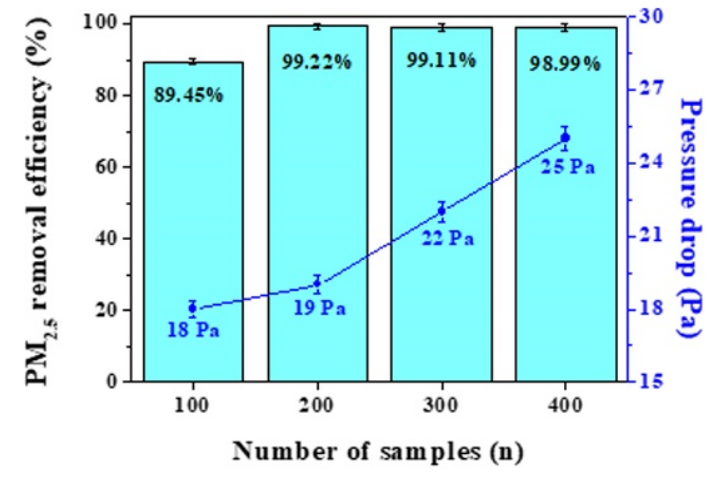

d)

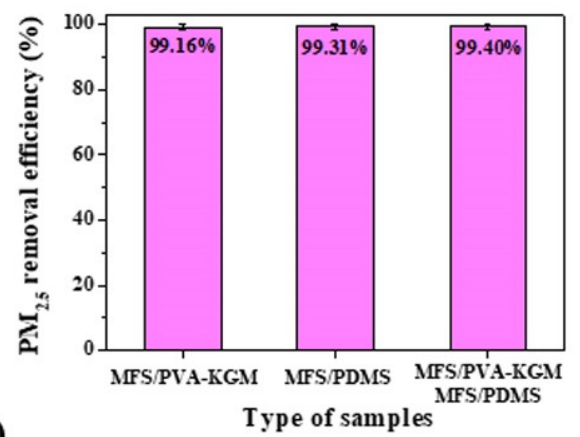

e)

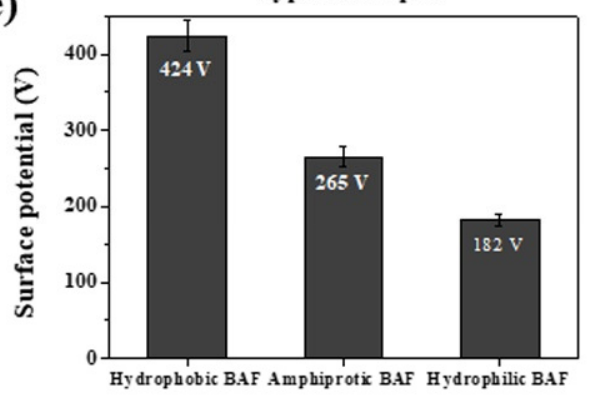

f)

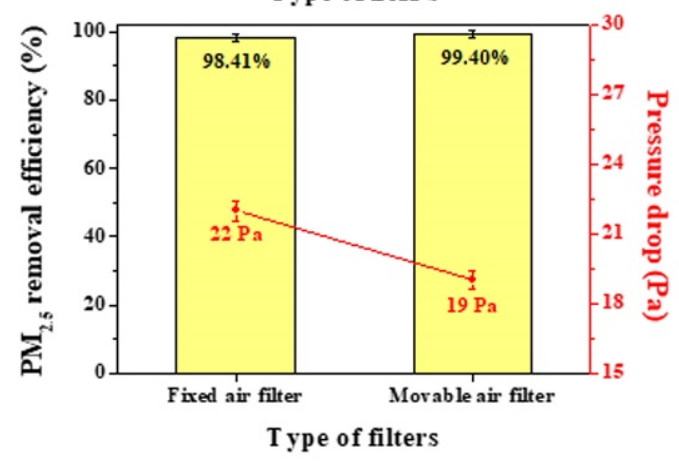

Figure 3. (a) An image of a tower air filtration system placed in an acrylate chamber. (b) An image of a tower air filtration system in which BAFs are actively rotated by a fan motor. (c) $\mathrm{PM}_{2.5}$ removal efficiencies and pressure drops of the air filtration systems loaded with hydrophilic BAF-100 to 400. (d) $\mathrm{PM}_{2.5}$ removal efficiencies of hydrophilic, hydrophobic, and hybrid BAF-200. (e) Surface potentials of hydrophilic, hydrophobic, and hybrid BAF-200. (f) $\mathrm{PM}_{2.5}$ removal efficiencies and pressure drops of the fixed BAFs (hybrid BAF-200) and movable BAFs (hybrid BAF-200).

To investigate the effect of the wettability of BAFs on the $\mathrm{PM}_{2.5}$ removal efficiency, the $\mathrm{PM}_{2.5}$ removal efficiency of hydrophobic and hybrid BAFs was measured and compared with that of hydrophilic BAFs. Hydrophobic BAF-200 (200 MFS/PDMS) and hybrid BAF200 (100 MFS/PVA-KGM + 100 MFS/PDMS) were used for exact comparison. Although the samples featured analogous $\mathrm{PM}_{2.5}$ removal efficiencies, the removal time of hybrid BAF-200 was remarkable (Figures 3d and S5b). The removal times were $6 \mathrm{~min}$ and $8 \mathrm{~min}$ for hybrid BAF-200 and hydrophobic BAF-200, respectively, which were faster and slower than that of hydrophilic BAF-200 (7 min) (Figure S5b). Based on the removal time (6 min), hybrid BAF-200 was the optimal BAF condition.

To reveal the reason why hybrid BAF-200 showed the best performance, the surface potentials of hydrophilic, hydrophobic, and hybrid BAF-200 were measured after the rotation of each BAF within the chamber for $5 \mathrm{~min}$. Hydrophobic BAF-200s exhibited 
the highest surface potential, of $424 \mathrm{~V}$, which was much higher than the values of $265 \mathrm{~V}$ and $182 \mathrm{~V}$ of hybrid BAF-200 and hydrophilic BAF-200, respectively (Figure 3e). The strongest electrostatic effect of hydrophobic BAF-200 was also observed with the naked eye after the rotation of each BAF within the chamber (hydrophobic BAF-200 > hybrid BAF-200 > hydrophilic BAF-200) (Figure S6). Through the vigorous rotation of hydrophobic BAF-200 within the glass chamber, a strong electric field can be generated through contact electrification. During the rotation of hydrophobic BAFs, the PDMS on hydrophobic BAFs generates negative charges, and the glass chamber forms positive charges according to the triboelectric series, leading to the formation of an electric field between the hydrophobic BAFs and the glass chamber. Since the PDMS of the hydrophobic BAFs and the glass of the chamber are at the opposite ends of the triboelectric series [29], hydrophobic BAFs can obtain electrons more easily than the others. The PVA of the hydrophilic BAFs is relatively close to the glass of the chamber in the triboelectric series [29], forming a weaker electric field than the PDMS of the hydrophobic BAFs. Thus, hydrophobic BAFs can efficiently eliminate PM using their strong electric fields. However, electric field by contact electrification may not be the main reason for hydrophilic BAFs because hydrophilic BAFs (MFS/PVA-KGM) possess a much lower surface potential (182 V) than hydrophobic BAFs $(424 \mathrm{~V})$.

The hydrophilic BAF (MFS/PVA-KGM) was composed of PVA and KGM, which feature high dipole moments (1.2 D and 3.1 D) [15,30]. These constituents can play an important role in removing PM because PM mainly consists of various hydrophilic components, such as sulfate, nitrate, silicate, calcium, chloride, and iron, and the polar groups of the constituents undergo strong dipole-dipole and induced-dipole interactions that can be used to capture PM [7,12]. Hybrid BAF-200 possessed both a high-dipole moment due to hydrophilic BAF-100 and a strong electric field due to hydrophobic BAF-100. Thus, PM can be removed by both effects. Hybrid BAF-200 showed analogous PM2.5 removal efficiency to the others (hybrid BAF-200 (99.4\%) = hydrophobic BAF-200 (99.3\%) = hydrophilic BAF-200 (99.1\%)). Hybrid BAF-200 showed slightly higher $\mathrm{PM}_{2.5}$ removal efficiency than the others (hybrid BAF-200 (99.4\%) > hydrophobic BAF-200 (99.3\%) > hydrophilic BAF-200 (99.1\%)), while it exhibited a much higher removal time performance than the others (hybrid BAF-200 $(6 \mathrm{~min})>$ hydrophilic BAF-200 $(7 \mathrm{~min})>$ hydrophobic BAF200 (8 min)) (Figures 3d and S5b). The $\mathrm{PM}_{2.5}$ removal efficiency (99.3\%) of hydrophobic BAF-200 was analogous to that of hydrophilic BAF-200 (99.1\%), while the removal time (7 min) of hydrophilic BAF-200 was shorter than that of hydrophobic BAF-200 (8 min) (Figures $3 \mathrm{~d}$ and S5b). The hydrophilic BAFs were dominant in the removal time. These results suggested that using a high dipole moment to remove PM was more suitable for lowering the removal time. Thus, the hybrid BAF, which exhibited the properties of both BAFs, offers both a fast removal time and a high $\mathrm{PM}_{2.5}$ removal efficiency.

To further reveal the characteristics of the hybrid BAFs, comparative experiments involving a movable hybrid BAF-200 and a fixed hybrid BAF-200 were conducted to test the removal of PM because the hybrid BAFs described here were movable. To test the fixed hybrid BAF, the hybrid BAF-200 was fixed at the bottom section of the air filter chamber (Figure S7). No movement of the hybrid BAFs was observed when PM-laden air passed through the air filter chamber. The $\mathrm{PM}_{2.5}$ removal efficiency was lower and the pressure drop of the fixed BAFs was higher than those of the movable BAFs, which indicated that the movable BAFs were advantageous over the fixed BAFs for the removal of PM (Figure 3f). The movable BAFs also removed the PM more quickly than the fixed BAFs (Figure S5c). The removal time to reach the $\mathrm{PM}_{2.5}$ concentration below $50 \mu \mathrm{g} / \mathrm{m}^{3}$ was $6 \mathrm{~min}$ for the movable BAFs, while it was 9 min for the fixed BAFs, suggesting that air filtration performances can depend on the filter type (movable or fixed BAF) even if hybrid BAFs are used in tandem. The fixed hydrophilic and hydrophobic BAFs further confirmed these results. The fixed hydrophilic and hydrophobic BAF-200 were also applied to test the removal of PM. The $\mathrm{PM}_{2.5}$ removal efficiencies of both fixed BAF-200 were lower than those of the movable BAF-200 (Figures 3d and S8). The fixed BAFs also removed the PM more slowly than 
the movable BAFs (Figures S5 and S8). Although hydrophobic BAFs showed the highest specific surface area among the samples (hydrophobic BAFs $\left(4.28 \mathrm{~m}^{2} / \mathrm{g}\right)>$ hybrid BAFs $\left(3.93 \mathrm{~m}^{2} / \mathrm{g}\right)>$ hydrophilic BAFs $\left.\left(3.58 \mathrm{~m}^{2} / \mathrm{g}\right)\right)$, the fixed hydrophobic BAFs showed the lowest $\mathrm{PM}_{2.5}$ removal efficiency (Figures S8 and S9). The Brunauer-Emmett-Teller (BET) data reconfirmed that air filtration performances can depend on the filter type (movable or fixed BAF) rather than specific surface areas. The abovementioned results demonstrated the superiority of the movable BAF over the fixed BAF.

To investigate the size effect of the BAF on the $\mathrm{PM}_{2.5}$ removal efficiency, an hybrid BAF with a diameter of $9 \mathrm{~mm}$ was prepared and compared with the hybrid BAF with a diameter of $4 \mathrm{~mm}$. Fewer $9 \mathrm{~mm}$-sized BAFs than $4 \mathrm{~mm}$-sized BAFs were used so that the total volume of the $9 \mathrm{~mm}$ BAFs was equivalent to that of the $4 \mathrm{~mm}$ BAFs. Although the total volume of the $9 \mathrm{~mm}$ BAFs was the same as that of the $4 \mathrm{~mm}$ BAFs, the $9 \mathrm{~mm}$ BAFs exhibited a lower $\mathrm{PM}_{2.5}$ removal efficiency and slower removal time than the $4 \mathrm{~mm}$ BAFs, which suggests that a small BAF is more favorable than a large BAF for the removal of PM due to its high mobility (Figure 4a). An hybrid BAF with a diameter below $4 \mathrm{~mm}$ was not tested because the $4 \mathrm{~mm}$-sized BAF was the minimum size that could be synthesized. To investigate the effect of the PM concentration on the $\mathrm{PM}_{2.5}$ removal efficiency, the removal performance of hybrid BAF-200 was tested at low PM concentrations (500 and $1000 \mu \mathrm{g} / \mathrm{m}^{3}$ ). As the PM concentrations decreased, the $\mathrm{PM}_{2.5}$ removal efficiencies decreased (Figure $4 \mathrm{~b}$ ). However, hybrid BAF-200 still showed a high $\mathrm{PM}_{2.5}$ removal efficiency of $98.5 \%$, even at a low concentration of $500 \mu \mathrm{g} / \mathrm{m}^{3}$ (Figure $4 \mathrm{~b}$ ). Extremely high $\mathrm{PM}_{2.5}$ concentrations of several tens of thousands to hundreds of thousands of $\mu \mathrm{g} / \mathrm{m}^{3}$ were used for air filtration tests in previous reports, although these conditions do not meet the requirements for application in real situations. Thus, air filters must be designed and developed to capture $\mathrm{PM}$ at very low concentrations (several tens to hundreds of $\mu \mathrm{g} / \mathrm{m}^{3}$ ).

a)

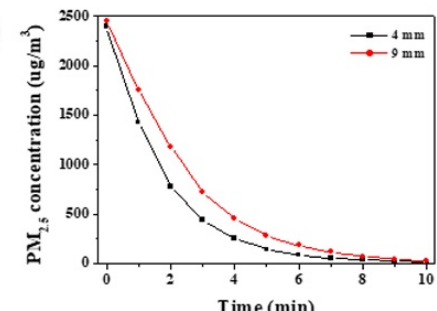

c)

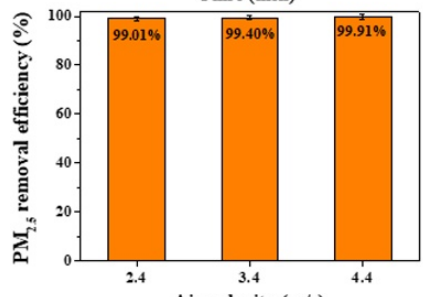

e)

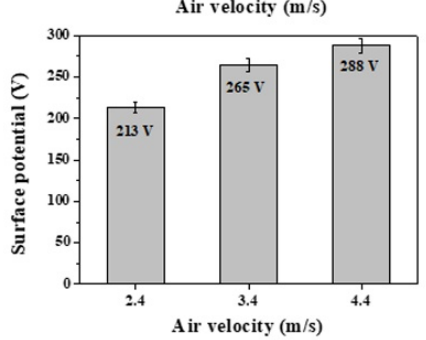

b)

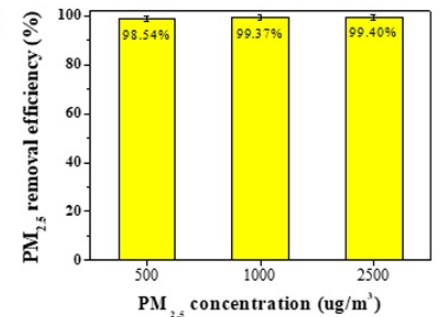

d)

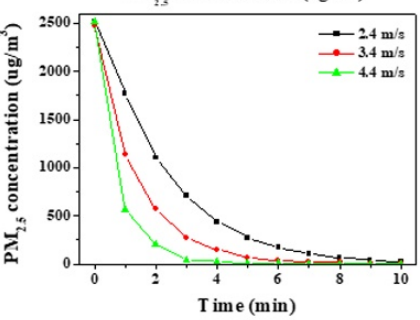

f)

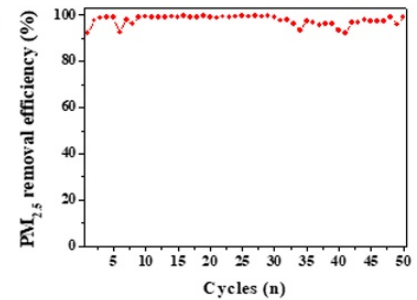

Figure 4. (a) $\mathrm{PM}_{2.5}$ removal efficiencies of $4 \mathrm{~mm}$ and $9 \mathrm{~mm}$ BAFs (hybrid BAF-200). (b,c) $\mathrm{PM}_{2.5}$ removal efficiencies of hybrid BAF-200 at different (b) $\mathrm{PM}_{2.5}$ concentrations $\left(500,1000\right.$, and $2500 \mu \mathrm{g} / \mathrm{m}^{3}$ ) and (c) air velocities $(2.4,3.4$, and $4.4 \mathrm{~m} / \mathrm{s})$. (d) $\mathrm{PM}_{2.5}$ concentration variations of hybrid BAF-200 at different air velocities $(2.4,3.4$, and $4.4 \mathrm{~m} / \mathrm{s})$. (e) Surface potentials of hybrid BAF-200 as a function of increasing air velocity $(2.4,3.4$, and $4.4 \mathrm{~m} / \mathrm{s}$ ). (f) Long-term performance of hybrid BAF-200 up to the 50th cycle. 
Since the air velocity was another important parameter affecting the PM removal efficiency, the $\mathrm{PM}_{2.5}$ removal efficiencies were measured as the air velocity increased. The PM removal efficiency generally decreased as the air velocity increased because of the decreased contact time between the PM and the air filter. However, the $\mathrm{PM}_{2.5}$ removal efficiencies of hybrid BAF-200 slightly increased to $99.9 \%$ at an air velocity of $4.4 \mathrm{~m} / \mathrm{s}$, which was slightly higher than $99.0 \%$ or $99.4 \%$ at air velocities of $2.4 \mathrm{~m} / \mathrm{s}$ and $3.4 \mathrm{~m} / \mathrm{s}$, respectively (Figure 4c). Against our expectations, the PM removal efficiency increased as the air velocity increased. The removal time to reach the $\mathrm{PM}_{2.5}$ concentration below $50 \mu \mathrm{g} / \mathrm{m}^{3}$ was $6 \mathrm{~min}$ at an air velocity of $3.4 \mathrm{~m} / \mathrm{s}$, while it was $3 \mathrm{~min}$ at an air velocity of $4.4 \mathrm{~m} / \mathrm{s}$ (Figure $4 \mathrm{~d}$ ). The abovementioned data suggest that the $\mathrm{PM}_{2.5}$ removal efficiency could be enhanced even at high air velocities. To further explore this phenomenon, the surface potentials of the hybrid BAF-200 were measured under different air velocities (2.4, 3.4 , and $4.4 \mathrm{~m} / \mathrm{s}$ ). The surface potential of hybrid BAF-200 was $213 \mathrm{~V}$ at an air velocity of $2.4 \mathrm{~m} / \mathrm{s}$, and it increased to $288 \mathrm{~V}$ at an air velocity of $4.4 \mathrm{~m} / \mathrm{s}$ (Figure $4 \mathrm{e}$ ). The surface potential gradually increased as the air velocity increased. At high air velocities, the chance of contact between PM and the BAFs is likely lower, while the chance of contact between the glass chamber and the BAFs is likely higher, leading to the formation of a stronger electric field and the removal of PM. It was speculated that the latter case was more common than the former case. The long-term performance of the hybrid BAF-200 was also tested for the practical application of the BAF. Each cycle was $10 \mathrm{~min}$, including the removal (8 $\mathrm{min})$ and stabilization $(2 \mathrm{~min})$ times. The operation time for the long-term performance test was $9 \mathrm{~h}$ with up to 50 cycles. Although the $\mathrm{PM}_{2.5}$ removal efficiencies of hybrid BAF-200 decreased to 95.9-93.4\% after several cycles, the $\mathrm{PM}_{2.5}$ removal efficiencies remained as high as 99.4-93.4\% until the 50th cycle (Figure 4f). In the absence of PMs, the weight variation of hybrid BAF-200 was monitored up to 50 cycles. After 50th cycle, the weight of hybrid BAF-200 slightly decreased to $98.8 \%$ (Figure S10).

The adsorption of PM onto the hybrid BAFs was confirmed by several measurements. Figure 5a,b shows SEM images of the BAFs before and after adsorption of PM. After filtration, aggregated particles were observed on the BAF surface (Figure 5b). The BAF surface became more complicated and rough after filtration. Fourier transform infrared (FT-IR) spectroscopy was performed to further confirm the adsorption of PM. After filtration, the peak intensities at $3289 \mathrm{~cm}^{-1}$ (-OH stretching), $1559 \mathrm{~cm}^{-1}\left(\mathrm{C}=\mathrm{C}\right.$ stretching), and $1443 \mathrm{~cm}^{-1}$ (C-N stretching) increased in the spectrum of the hydrophilic BAF (MFS/PVA-KGM), which indicated the adsorption of PM onto the hydrophilic BAFs because polar functional groups and elemental carbons were present at the outer surface of the PM (Figure 5c, red line) [12]. However, the peak intensities at $1084 \mathrm{~cm}^{-1}$ (C-O stretching) and $808 \mathrm{~cm}^{-1}$ (C-O-C stretching) in the spectrum of the hydrophilic BAF were lower, which suggested the adsorption of PM onto the C-O or C-O-C group of the hydrophilic BAF (Figure 5c, red line) [12]. The characteristic peaks of PDMS were lower in the spectrum of the hydrophobic BAF (MFS/PDMS) at $2961 \mathrm{~cm}^{-1}$ (-CH stretching), $1258 \mathrm{~cm}^{-1}\left(\mathrm{Si}-\mathrm{CH}_{3}\right.$ stretching), $1016 \mathrm{~cm}^{-1}$ (Si-O-Si stretching), and $793 \mathrm{~cm}^{-1}\left(\mathrm{Si}-\mathrm{CH}_{3}\right.$ stretching), confirming the adsorption of PM onto the hydrophobic BAFs (Figure 5d, blue line) [23-25]. The amount of PM adsorbed onto the hydrophilic and hydrophobic regions of the hybrid BAFs was measured by thermogravimetric analysis (TGA). The hydrophilic BAFs possessed $6.44 \%$ more mass after filtration than before filtration, while the hydrophobic BAFs possessed $1.5 \%$ more mass after filtration than before filtration, further confirming the adsorption of PM onto both hydrophilic and hydrophobic BAFs (Figure 5e,f). These TGA data also confirmed the greater adsorption of PM onto the hydrophilic BAFs than onto the hydrophobic BAFs. However, the $\mathrm{PM}_{2.5}$ removal efficiencies of hydrophilic and hydrophobic BAFs were $99.1 \%$ and $99.3 \%$, respectively, which were similar to each other. These results suggested that stronger dipole-dipole and induced-dipole interactions were the driving forces of PM capture by the hydrophilic BAFs because the detected PM was mainly adsorbed on the hydrophilic BAFs. However, a strong electric field was the main cause of PM removal from the hydrophobic BAFs because the adsorption of PM was mainly observed at the 
inside surface of the glass chamber rather than hydrophobic BAFs. These results were in agreement with the previous surface potential data.
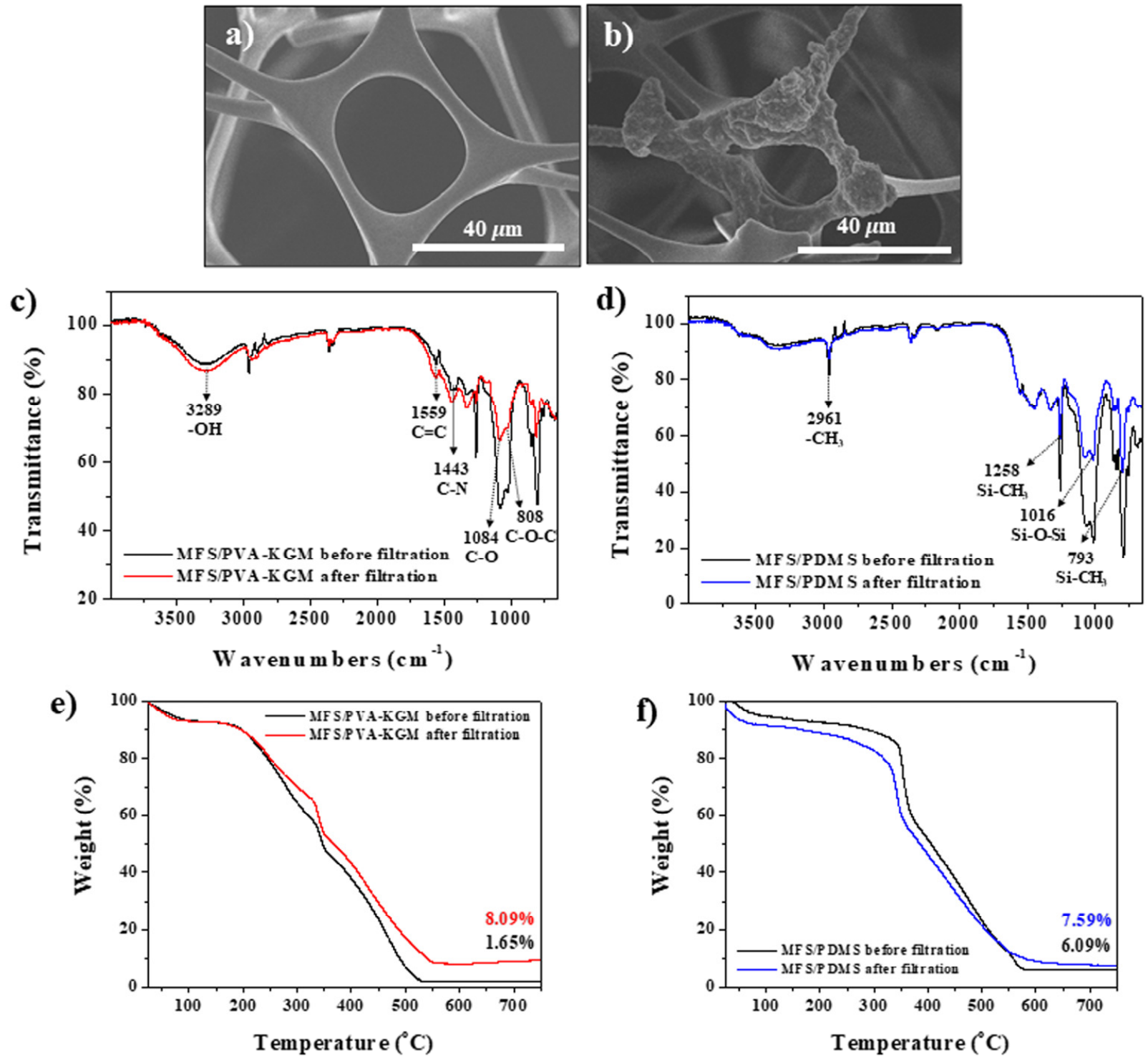

Figure 5. SEM images of BAFs (a) before and (b) after adsorption of PM. (c,d) FT-IR and (e,f) TGA data of $(\mathbf{c}, \mathbf{e})$ hydrophilic and $(\mathbf{d}, \mathbf{f})$ hydrophobic BAFs before and after PM filtration.

To investigate the removal ability of HCHO by hydrophilic BAF-200, a tower air filtration system loaded with hydrophilic BAF-200 was placed in a chamber $(3.63 \mathrm{~L})$ containing a $\mathrm{HCHO}$ concentration of $4.87 \mathrm{ppm}$ (Figure 6a). Since the hydroxyl groups in the adsorbent can enhance the adsorption affinity of HCHO [31], hydrophilic BAFs (MFS/PVA-KGM) were tested. To further increase the adsorption affinity, two types of hydrophilic BAF (MFS/PVA-KGM, KGM-9\% and 33\%) were used and compared. Hydrophilic BAFs containing KGM contents over 33\% were not tested because they showed poor durability as the KGM content increased. KGM contains 11 hydroxyl groups per repeating unit (Figure 1a). The variation in the $\mathrm{HCHO}$ gas concentration emitted from the chamber with and without the air filter was monitored as time increased. The fan was operated even in the absence of an air filter for exact comparison. In the absence of the air filter, the concentration of $\mathrm{HCHO}$ gas was $0.7 \mathrm{ppm}$ at $60 \mathrm{~min}$ (black line), while it was 0.48 and $0.41 \mathrm{ppm}$ at $60 \mathrm{~min}$ in the presence of hydrophilic BAF (KGM-9\%) and BAF (KGM-33\%), respectively (red and green lines, Figure 6b). Against our expectations, at $130 \mathrm{~min}$, the concentrations of $\mathrm{HCHO}$ 
gas remarkably increased to $0.92 \mathrm{ppm}$ when no filter was used and slightly increased to 0.54 ppm with hydrophilic BAF (KGM-9\%) (black and red lines, respectively). However, the concentration of $\mathrm{HCHO}$ gas further decreased to $0.31 \mathrm{ppm}$ with the hydrophilic BAF (KGM-33\%) (green line). Thus, the net removal efficiency of the hydrophilic BAF (KGM$33 \%$ ) was $66 \%$ at $130 \mathrm{~min}$ (Figure S11). The net removal capacities of the hydrophilic BAF (KGM-33\%) and BAF (KGM-9\%) for $\mathrm{HCHO}$ were 9.54 and $5.94 \mathrm{mg} \mathrm{g}^{-1}$, respectively; these were calculated based on the concentration ratios of the $\mathrm{HCHO}$ at $130 \mathrm{~min}$ (Figure S11). When no filter was used, $\mathrm{HCHO}$ could desorb from the surface of the acrylate chamber because $\mathrm{HCHO}$ physically adsorbed onto the inside surface of the acrylate chamber (Figure 6b, black line). A relatively low desorption of $\mathrm{HCHO}$ gas was observed in the hydrophilic BAF (KGM-9\%) (Figure 6b, red line). Since the hydrophilic BAF (KGM-9\%) consists of KGM containing 11 hydroxyl groups per repeating unit, the low desorption of HCHO gas could be attributed to the abundant hydroxyl groups in the hydrophilic BAF (KGM-9\%), which was further confirmed by the hydrophilic BAF (KGM-33\%) case. No desorption of $\mathrm{HCHO}$ gas was observed in the hydrophilic BAF (KGM-33\%), while additional adsorption of $\mathrm{HCHO}$ gas was observed. These results indicated that the hydrophilic BAF (KGM-33\%), which possessed more abundant hydroxyl groups than the hydrophilic BAF (KGM-9\%), could stably and tightly capture more $\mathrm{HCHO}$ gas without any desorption of HCHO gas.

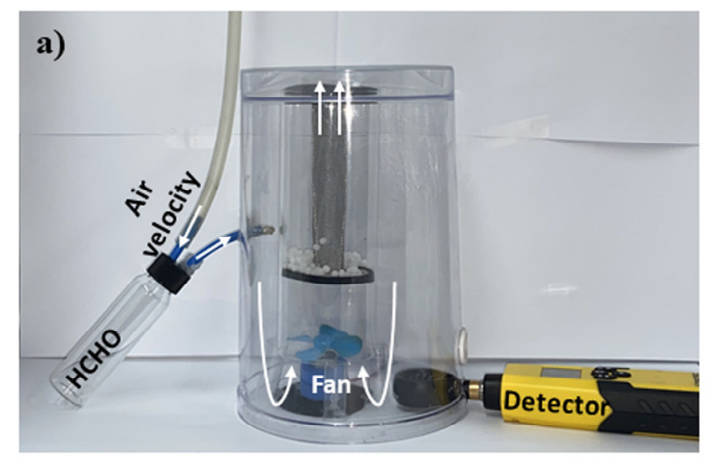

b)

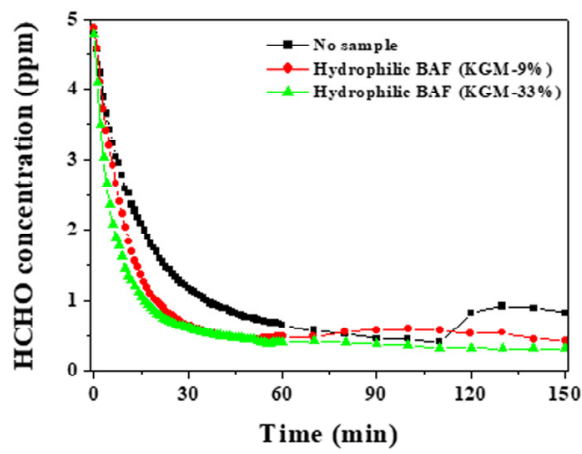

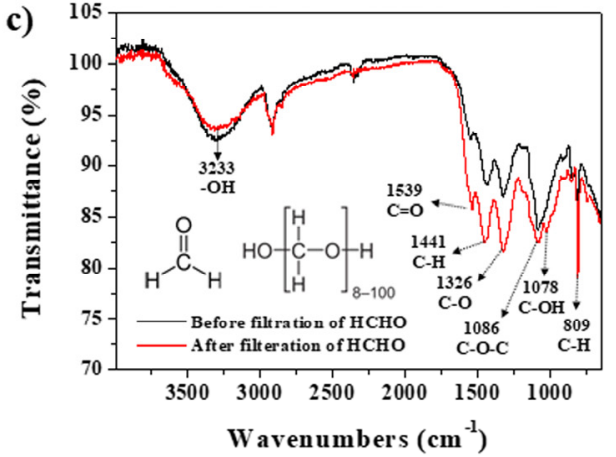

Figure 6. (a) An image showing a tower air filtration system loaded with hydrophilic BAF-200 for the removal of HCHO. (b) HCHO concentration variations of hydrophilic BAF-200 (KGM-9 and 33\%) for $150 \mathrm{~min}$. (c) FT-IR data of hydrophilic BAF-200 (KGM-33\%) before and after HCHO filtration.

$\mathrm{HCHO}$ gas could be polymerized to para-HCHO due to its instability when $\mathrm{HCHO}$ gas was adsorbed onto the hydroxyl groups of hydrophilic BAF [32,33]. The para-HCHO could not desorb from the surface of the hydrophilic BAF due to the size of the para-HCHO, even when pumped under vacuum at room temperature. These results suggest that the $\mathrm{HCHO}$ adsorbed on the hydrophilic BAF would not desorb during daily use. According to the guidelines of the World Health Organization (WHO) [34], the occupational exposure limit of $\mathrm{HCHO}$ gas is $0.5 \mathrm{ppm}$ per $8 \mathrm{~h}$. Hydrophilic BAF (KGM-33\%) maintains $\mathrm{HCHO}$ concentrations of $0.41-0.31 \mathrm{ppm}$ at $60-150 \mathrm{~min}$, which is below the WHO guidelines (Figure 6b, green line). However, in the absence of an air filter, the concentrations of 
HCHO gas were 0.7-0.92 ppm even under strong air circulation, which exceeded the WHO guidelines (Figure $6 \mathrm{~b}$, black line). Previous reports did not meet the needs of real situations because extremely high $\mathrm{HCHO}$ concentrations of several hundred to a thousand ppm are generally used for $\mathrm{HCHO}$ adsorption tests $[32,33]$. The abovementioned results demonstrated that hydrophilic BAF (KGM-33\%) was useful for practical concentration ranges of $\mathrm{HCHO}$ exposure $(<5 \mathrm{ppm})$ in daily life. To confirm the adsorption of $\mathrm{HCHO}$ gas onto the hydrophilic BAF (KGM-33\%), FT-IR spectroscopy was performed. The decrease in the $-\mathrm{OH}$ stretching band at $3233 \mathrm{~cm}^{-1}$ suggested the adsorption of $\mathrm{HCHO}$ onto the hydroxyl group of the hydrophilic BAF (red line) [15,32,33]. Six vibrational bands at $1539 \mathrm{~cm}^{-1}$ (C=O stretching), $1441 \mathrm{~cm}^{-1}$ (-CH stretching), $1326 \mathrm{~cm}^{-1}$ (C-O stretching), $1086 \mathrm{~cm}^{-1}$ (C-O-C stretching), $1078 \mathrm{~cm}^{-1}$ (C-OH stretching), and $809 \mathrm{~cm}^{-1}$ (CH stretching), increased in intensity and were attributed to the formation of para- $\mathrm{HCHO}$ (Figure $6 \mathrm{c}$, red line) $[15,32,33]$. FT-IR data demonstrated transformation of the $\mathrm{HCHO}$ gas into para-HCHO in the hydrophilic BAF.

\section{Conclusions}

Hydrophilic and hydrophobic BAFs were synthesized through the physical etching of a cylindrical MFS, followed by one-step coating of the MFS bead with PVA-KGM and PDMS, respectively. Hybrid BAFs were prepared by combining hydrophilic and hydrophobic BAFs. A tower air filtration system in which BAFs were actively rotated by a fan motor to remove $\mathrm{PM}$ or $\mathrm{HCHO}$ gas was designed. A tower air filtration system loaded with the hybrid BAF-200 purified $3.63 \mathrm{~L}$ of PM $\left(2500 \mu \mathrm{g} / \mathrm{m}^{3} \mathrm{PM}_{2.5}\right)$ at a high flow rate of $3.4 \mathrm{~m} / \mathrm{s}$ with high removal efficiency $\left(99.4 \%\right.$ for $\mathrm{PM}_{2.5}$ ) and a low pressure drop (19 Pa) over $6 \mathrm{~min}$. Since hybrid BAF-200 possessed both a high-dipole moment due to the hydrophilic BAF-100 and a strong electric field due to the hydrophobic BAF-100, PM was effectively removed by both substituents. The pressure drop (19 Pa) was much lower than those of previously reported filters, even at a high air velocity of an order of magnitude higher than those previously reported. Against our expectations, the $\mathrm{PM}_{2.5}$ removal efficiency slightly increased as the air velocity increased, suggesting that the removal efficiency could be enhanced even at high air velocities because the chance of contact between the glass chamber and the BAFs increased, leading to the formation of a stronger electric field. The hybrid BAF-200 showed excellent recyclability of up to 50 cycles with high removal efficiencies (99.4-93.4\% for $\mathrm{PM}_{2.5}$ ). Furthermore, hydrophilic BAF-200 could permanently remove $3.73 \mathrm{~L}$ of $\mathrm{HCHO}$ gas (4.87 ppm) and return the ambient HCHO to safe levels (0.41-0.31 ppm) within $60 \mathrm{~min}$ without any desorption of $\mathrm{HCHO}$ gas.

Supplementary Materials: The following are available online at https:/ / www.mdpi.com/article/10 .3390 / polym14030422/s1. Figure S1: Images of $4 \mathrm{~mm}$ - and $9 \mathrm{~mm}$-sized bead MFS sponges. Figure S2: (Top) SEM Image of MFS and (bottom) its corresponding EDX data. Figure S3: Image of a tower air filtration system equipped with a differential pressure gauge. Figure S4: Images of air filtration systems loaded with (a) BAF-300 and (b) BAF-400. Figure S5: PM $_{2.5}$ concentration variations of hydrophilic BAF-100 to 400 as a function of increasing time. $\mathrm{PM}_{2.5}$ concentration variations of hydrophilic, hydrophobic, and hybrid BAF-200 as a function of increasing time. $\mathrm{PM}_{2.5}$ concentration variations of the fixed BAFs (hybrid BAF-200) and movable BAFs (hybrid BAF-200) as a function of increasing time. Figure S6: Images of hybrid BAF-200 and hydrophobic BAF-200 after operation. Figure S7: Image of the hybrid BAF-200 fixed at the bottom section of the air filter chamber. Figure S8: (a) PM2.5 removal efficiencies of fixed air filters. (b) PM2.5 concentration variations of fixed air filters as a function of increasing time. Figure S9: (a) BET nitrogen adsorption-desorption isotherm of hydrophilic BAFs (MFS/PVA-KGM) and hydrophobic BAFs (MFS/PDMS). (b) BET surface areas of hydrophilic BAFs, hydrophobic BAFs, and hybrid BAFs. Figure S10: Weight variations of hybrid BAF200 up to 50 cycles. Figure S11: Net HCHO removal (a) efficiencies and (b) capacities of hydrophilic BAF (KGM-33\%) and BAF (KGM-9\%). 


\begin{abstract}
Author Contributions: Conceptualization, W.S.C.; methodology, H.J.K., Y.J.K., Y.J.S., J.H.C. and H.Y.K.; validation, W.S.C. and H.J.K.; investigation, H.J.K., Y.J.K., Y.J.S. and J.H.C.; writing—original draft preparation, H.J.K.; writing—review and editing, W.S.C. and H.Y.K.; supervision, W.S.C. All authors have read and agreed to the published version of the manuscript.
\end{abstract}

Funding: This research received no external funding.

Institutional Review Board Statement: Not applicable.

Informed Consent Statement: Not applicable.

Data Availability Statement: Not applicable.

Acknowledgments: This research was supported by the research fund of Hanbat National University in 2021.

Conflicts of Interest: The authors declare no conflict of interest.

\title{
References
}

1. Liu, H.; Cao, C.; Huang, J.; Chen, Z.; Chen, G.; Lai, Y. Progress on particulate matter filtration technology: Basic concepts, advanced materials, and performances. Nanoscale 2020, 12, 437-453. [CrossRef]

2. Souzandeh, H.; Wang, Y.; Netravali, A.N.; Zhong, W.H. Towards Sustainable and Multifunctional Air Filters: A Review on Biopolymer-Based Filtration Materials. Polym. Rev. 2019, 59, 651-686. [CrossRef]

3. Liu, G.; Xiao, M.; Zhang, X.; Gal, C.; Chen, X.; Liu, L.; Pan, S.; Wu, J.; Tang, L.; Clements-Croome, D. A review of air filtration technologies for sustainable and healthy building ventilation. Sustain. Cities Soc. 2017, 32, 375-396. [CrossRef]

4. Wu, J.; Wang, N.; Zhao, Y.; Jiang, L. Electrospinning of multilevel structured functional micro-/nanofibers and their applications. J. Mater. Chem. A 2013, 1, 7290-7305. [CrossRef]

5. Han, N.; Lee, Y.S.; Kaang, B.K.; Jang, W.; Koo, H.Y.; Choi, W.S. A lottery draw machine-inspired movable air filter with high removal efficiency and low pressure drop at a high flow rate. J. Mater. Chem. A 2019, 7, 6001-6011. [CrossRef]

6. Chen, Y.; Zhang, S.; Cao, S.; Li, S.; Chen, F.; Yuan, S.; Xu, C.; Zhou, J.; Feng, X.; Ma, X.; et al. Roll-to-roll production of metal-organic framework coatings for particulate matter removal. Adv. Mater. 2017, 29, 1606221. [CrossRef]

7. Jeong, S.; Cho, H.; Han, S.; Won, P.; Lee, H.; Hong, S.; Yeo, J.; Kwon, J.; Ko, S.H. High Efficiency, Transparent, Reusable, and Active PM2.5 Filters by Hierarchical Ag Nanowire Percolation Network. Nano Lett. 2017, 17, 4339-4346. [PubMed]

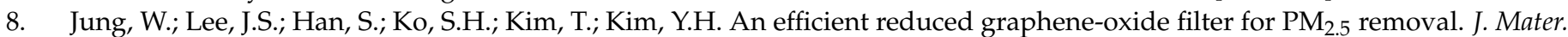
Chem. A 2018, 6, 16975-16982. [CrossRef]

9. Bai, Y.; Han, C.B.; He, C.; Gu, G.Q.; Nie, J.H.; Shao, J.J.; Xiao, T.X.; Deng, C.R.; Wang, Z.L. Washable Multilayer Triboelectric Air Filter for Efficient Particulate Matter $\mathrm{PM}_{2.5}$ Removal. Adv. Funct. Mater. 2018, 28, 1706680. [CrossRef]

10. Iranpour, R.; Cox, H.H.J.; Deshusses, M.A.; Schroeder, E.D. Literature review of air pollution control biofilters and biotrickling filters for odor and volatile organic compound removal. Environ. Prog. 2005, 24, 254-267. [CrossRef]

11. Zeng, Z.; Ma, X.Y.D.; Zhang, Y.; Wang, Z.; Ng, B.F.; Wan, M.P.; Lu, X. Robust lignin- based aerogel filters: High-efficiency capture of ultrafine airborne particulates and the mechanism. ACS Sustain. Chem. Eng. 2019, 7, 6959-6968. [CrossRef]

12. Liu, C.; Hsu, P.C.; Lee, H.W.; Ye, M.; Zheng, G.; Liu, N.; Li, W.; Cui, Y. Transparent air filter for high-efficiency PM 2.5 capture. Nat. Commun. 2015, 6, 6205. [CrossRef]

13. Lee, H.J.; Choi, W.S. 2D and 3D Bulk Materials for Environmental Remediation: Air Filtration and Oil/Water Separation. Materials 2020, 13, 5714. [CrossRef]

14. Zhang, S.; Liu, H.; Yu, J.; Luo, W.; Ding, B. Microwave structured polyamide-6 nanofiber/net membrane with embedded poly (m-phenylene isophthalamide) staple fibers for effective ultrafine particle filtration. J. Mater. Chem. A 2016, 4, 6149-6157.

15. Kaang, B.K.; Lee, H.B.; Koo, H.Y.; Choi, W.S. A wastepaper-based cylindrical hollow air filter module for the removal of particulate matter $\left(\mathrm{PM}_{10}\right.$ and $\left.\mathrm{PM}_{2.5}\right)$ and HCHO. ACS Sustain. Chem. Eng. 2020, 8, 13984-13996. [CrossRef]

16. Park, S.; Koo, H.Y.; Yu, C.; Choi, W.S. A novel approach to designing air filters: Ubiquitous material-based Janus air filter modules with hydrophilic and hydrophobic parts. Chem. Eng. J. 2021, 410, 128302-128311. [CrossRef]

17. Anttila, A.; Apostoli, P.; Bond, J.A.; Gerhardsson, L.; Gulson, B.L.; Hartwig, A.; Hoet, P.; Ikeda, M.; Jaffe, E.K.; Landrigan, P.J.; et al. IARC Monographs on the Evaluation of Carcinogenic Risks to Humans: Inorganic and Organic Lead Compounds; World Health Organization International Agency for Research on Cancer: Lion, France, 2006; Volume 87, pp. 217-375.

18. Gałęzowska, G.; Chraniuk, M.; Wolska, L. In vitro assays as a tool for determination of VOCs toxic effect on respiratory system: A critical review. Trend Anal. Chem. 2016, 77, 14-22. [CrossRef]

19. Kamal, M.S.; Razzak, S.A.; Hossain, M.M. Catalytic oxidation of volatile organic compounds (VOCs)—A review. Atmos. Environ. 2016, 140, 117-134. [CrossRef]

20. Niu, J.; Liland, S.E.; Yang, J.; Rout, K.R.; Ran, J.; Chen, D. Effect of oxide additives on the hydrotalcite derived Ni catalysts for $\mathrm{CO}_{2}$ reforming of methane. Chem. Eng. J. 2019, 377, 119763. [CrossRef]

21. Lan, Y.; Yang, Z.; Wang, P.; Yan, Y.; Zhang, L.; Ran, J. A review of microscopic seepage mechanism for shale gas extracted by supercritical $\mathrm{CO}_{2}$ flooding. Fuel 2019, 238, 412-424. [CrossRef] 
22. Niu, J.; Du, X.; Ran, J.; Wang, R. Dry $\left(\mathrm{CO}_{2}\right)$ reforming of methane over Pt catalysts studied by DFT and kinetic modeling. Appl. Surf. Sci. 2016, 376, 79-90. [CrossRef]

23. Lee, Y.S.; Lee, H.B.; Koo, H.Y.; Choi, W.S. Remote-Controlled Magnetic Sponge Balls and Threads for Oil/Water Separation in a Confined Space and Anaerobic Reactions. ACS Appl. Mater. Interfaces 2019, 11, 40886-40897.

24. Lee, Y.S.; Lim, Y.T.; Choi, W.S. One-step synthesis of environmentally friendly superhydrophilic and superhydrophobic sponges for oil/water separation. Materials 2019, 12, 1182. [CrossRef] [PubMed]

25. Socrates, G. Infrared Characteristic Group Frequencies, Tables and Charts. J. Am. Chem. Soc. 1995, $117,1671$.

26. Wang, N.; Raza, A.; Si, Y.; Yu, J.; Sun, G.; Ding, B. Tortuously structured polyvinyl chloride/polyurethane fibrous membranes for high-efficiency fine particulate filtration. J. Colloid Interface Sci. 2013, 398, 240-246. [CrossRef]

27. Zhang, R.; Liu, C.; Hsu, P.C.; Zhang, C.; Liu, N.; Zhang, J.; Lee, H.R.; Lu, Y.; Qiu, Y.; Chu, S.; et al. Nanofiber Air Filters with High-Temperature Stability for Efficient $\mathrm{PM}_{2.5}$ Removal from the Pollution Sources. Nano Lett. 2016, 16, 3642-3649. [CrossRef]

28. Zhao, X.; Li, Y.; Hua, T.; Jiang, P.; Yin, X.; Yu, J.; Ding, B. Low-Resistance Dual-Purpose Air Filter Releasing Negative Ions and Effectively Capturing PM2. ACS Appl. Mater. Interfaces 2017, 9, 12054-12063. [CrossRef]

29. Henniker, J. Triboelectricity in Polymers. Nature 1962, 196, 474. [CrossRef]

30. Ibrahim, H.S.; Ammar, N.S.; Soylak, M.; Ibrahim, M. Removal of Cd (II) and Pb (II) from aqueous solution using dried water hyacinth as a biosorbent. Spectrochim. Acta Part A 2012, 96, 413-420. [CrossRef]

31. Li, X.; Zhang, L.; Yang, Z.; Wang, P.; Yan, Y.; Ran, J. Adsorption materials for volatile organic compounds (VOCs) and the key factors for VOCs adsorption process: A review. Sep. Purif. Technol. 2020, 235, 116213. [CrossRef]

32. Srisuda, S.; Virote, B. Adsorption of formaldehyde vapor by amine-functionalized mesoporous silica materials. J. Environ. Sci. 2008, 20, 379-384. [CrossRef]

33. Chi, M.; Zhao, Y.P. Adsorption of formaldehyde molecule on the intrinsic and Al-doped graphene: A first principle study. Comp. Mater. Sci. 2009, 46, 1085-1090. [CrossRef]

34. Salthammer, T. The formaldehyde dilemma. Int. J. Hyg. Environ. Health 2015, 218, 433-436. [CrossRef] [PubMed] 